\title{
FERROELECTRIC FIELD-EFFECT TRANSISTOR DIFFERENTIAL AMPLIFIER CIRCUIT ANALYSIS
}

\author{
THOMAS A. PHILLIPS ${ }^{\mathrm{a}}$, TODD C. MACLEOD ${ }^{\mathrm{a}}$, and FAT D. HO ${ }^{\mathrm{b}}$ \\ ${ }^{\mathrm{a}}$ National Aeronautics and Space Administration, Marshall Space Flight Center, \\ Huntsville, Alabama, 35812, U.S.A. \\ ${ }^{\mathrm{b}}$ The University of Alabama in Huntsville, Department of Electrical and Computer Engineering, \\ Huntsville, Alabama 35899, U.S.A.
}

\begin{abstract}
There has been considerable research investigating the Ferroelectric Field-Effect Transistor (FeFET) in memory circuits. However, very little research has been performed in applying the FeFET to analog circuits. This paper investigates the use of FeFETs in a common analog circuit, the differential amplifier. The two input Metal-Oxide-Semiconductor (MOS) transistors in a general MOS differential amplifier circuit are replaced with FeFETs. Resistors are used in place of the other three MOS transistors. The FeFET model used in the analysis has been previously reported and was based on experimental device data. Because of the FeFET hysteresis, the FeFET differential amplifier has four different operating modes depending on whether the FeFETs are positively or negatively polarized. The FeFET differential amplifier operation in the different modes was analyzed by calculating the amplifier voltage transfer and gain characteristics shown in figures 2 through 5 . Comparisons were made between the FeFET differential amplifier and the standard MOS differential amplifier. Possible applications and benefits of the FeFET differential amplifier are discussed.
\end{abstract}




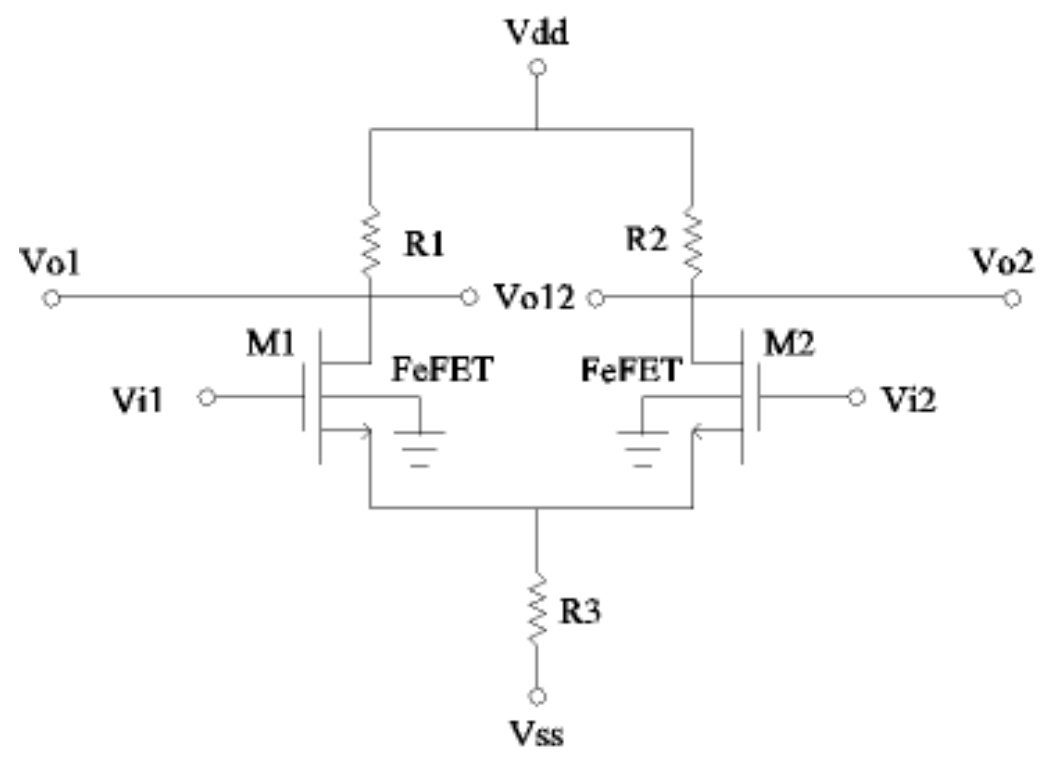

Figure 1: FeFET Differential Amplifier Circuit

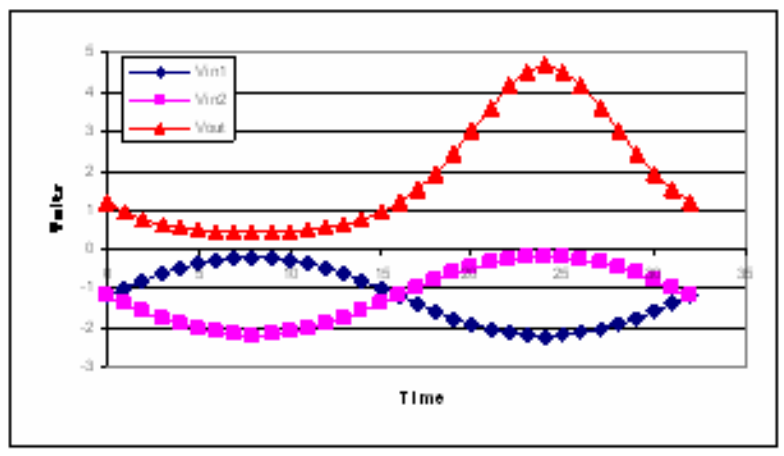

Figure 2: FeFET Diff. Amp.Voltage Transfer at -1.2V

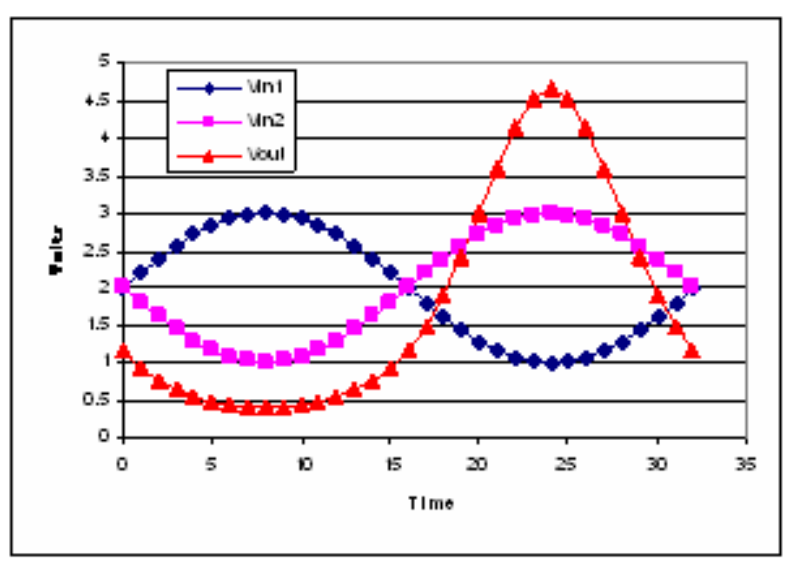

Figure 3: FeFET Diff. Amplifier Voltage Transfer at 2V

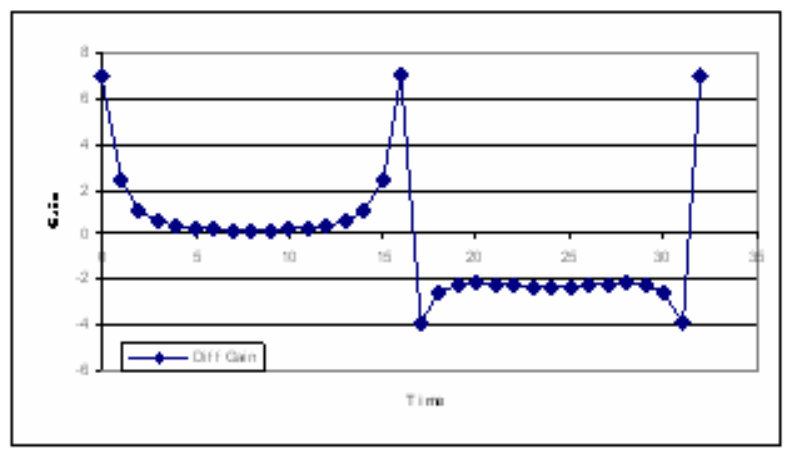

Figure 4: FeFET Differential Amplifier Gain vs. Time

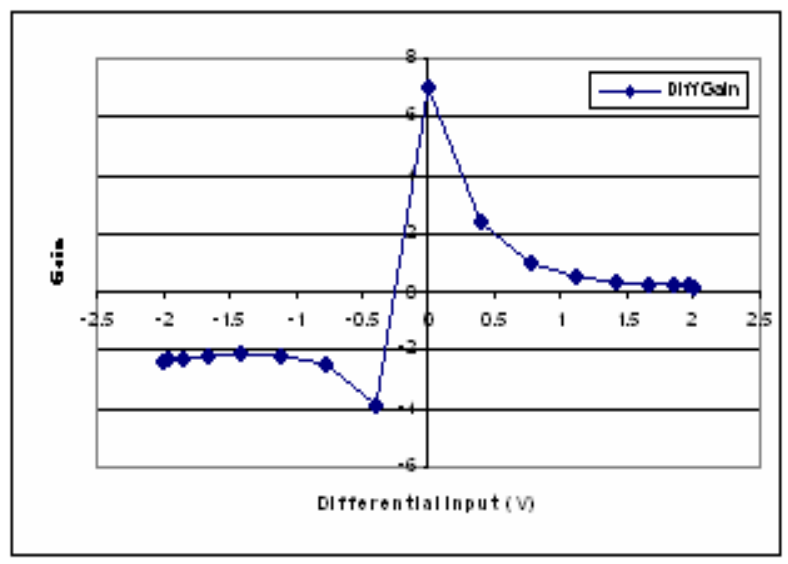

Figure 5: FeFET Differential Amplifier Gain vs. Input 


\title{
FERROELECTRIC FIELD-EFFECT TRANSISTOR DIFFERENTIAL AMPLIFIER CIRCUIT ANALYSIS
}

\author{
THOMAS A. PHILLIPSa, TODD C. MACLEODa, and FAT D. HO
}

aNational Aeronautics and Space Administration, Marshall Space Flight Center, Huntsville, Alabama, 35812, U.S.A.

${ }^{\mathrm{b}}$ The University of Alabama in Huntsville, Department of Electrical and Computer Engineering, Huntsville, Alabama 35899, U.S.A.

\section{INTRODUCTION}

- There has been considerable research investigating the Ferroelectric Field-Effect Transistor (FeFET) in memory circuits.

- However, very little research has been performed in applying FeFETs to analog circuits.

- This paper investigates the use of FeFETs in a common analog circuit, the differential amplifier.

- Goal to determine feasibility of using the FeFETs in the differential amplifier circuit.

\section{FERROELECTRIC TRANSISTOR}

- Initially the FeFET 4 × 400 transistors were characterized.

- Channel length $=4 \mu \mathrm{m}$

- $\quad$ Channel width $=400 \mu \mathrm{m}$

- FeFET active and remanent experimental data is shown in Figures 1 and 2 , respectively. [1]

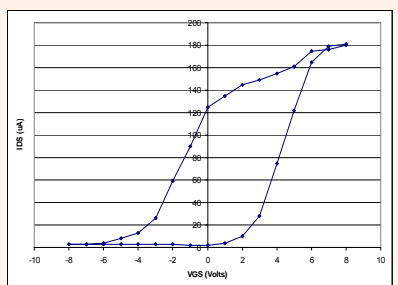

Figure 1: Active (On) Mode

acquisition limitations. [2]

\section{FERROELECTRIC DIFFERENTIAL AMPLIFIER}

- The FeFET differential amplifier circuit is shown in Figure 3. [3]

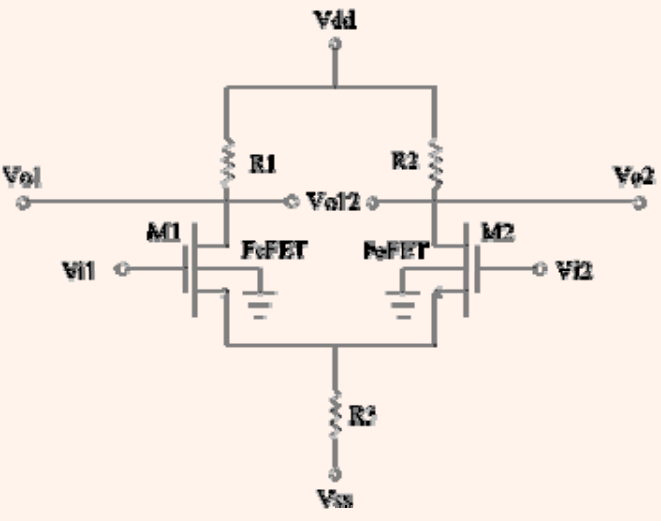

Figure 3: FeFET Differential Amplifier Circuit

- The two input Metal-Oxide-Semiconductor (MOS) transistors in a general MOS differential amplifier circuit are replace with $4 \times 400$ FeFETs M1 and M2.

- $400 \mathrm{~K} \Omega$ resistors R1, R2, and R3 are used in place of the other three MOS transistors.

- Because of the FeFET hysteresis, the differential amplifier has four different operating modes depending on whether M1 and M2 are positively or negatively polarized.

\section{EXPERIMENTAL RESULTS}

- Oscilloscope plots for various amplifier inputs are shown in Figures 4 through $7 . V_{i 1}$ and $V_{i 2}$ are the blue and yellow traces and $V_{0}$ is the red

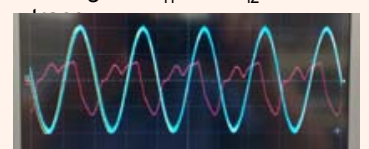

Figure 4: $\mathrm{V}_{\text {in }} @ 100 \mathrm{~Hz}, 8 \mathrm{~V}_{\mathrm{p}-\mathrm{p}}$ $0^{\circ}$ phase difference. $\mathrm{V}_{\mathrm{O} 1}$ is shown.

Figure 6: $\mathrm{V}_{\text {in }} @ 1 \mathrm{MHz}, 4 \mathrm{~V}$ $180^{\circ}$ phase difference. $V_{\mathrm{O} 2}$ is shown.

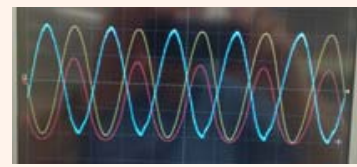

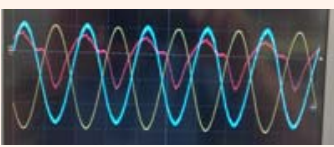

Figure 5: $\mathrm{V}_{\text {in }} @ 100 \mathrm{~Hz}, 4 \mathrm{~V}_{\mathrm{p}-\mathrm{p}}$, $180^{\circ}$ phase diff., $-1.0 \mathrm{Vdc}$ offset. $\mathrm{V}_{\mathrm{O} 2}$ is shown.

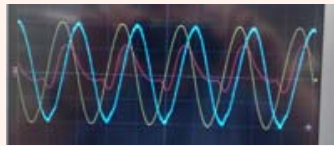

Figure 7: $\mathrm{V}_{\text {in }} @ 1 \mathrm{MHz}, 8 \mathrm{~V}_{\mathrm{p}-\mathrm{p}}$, $90^{\circ}$ phase difference. $\mathrm{V}_{\mathrm{O} 2}$ is shown.
- Comparison of FeFET diff. amp. to a standard MOSFET diff. amp. using MPF102 transistors is shown in Figures 8 through 11. $V_{i 1}$ and $V_{i 2}$ are the blue and yellow traces, $\mathrm{V}_{\mathrm{O} 1}$ is the green trace, $\mathrm{V}_{\mathrm{O} 2}$ is the pink trace, and

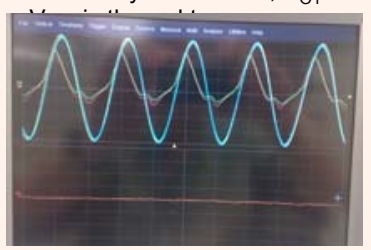

Figure 8: $\mathrm{V}_{\text {in }} @ 1 \mathrm{KHz}, 16 \mathrm{~V}_{\mathrm{p}-\mathrm{p}}$, $0^{\circ}$ phase difference

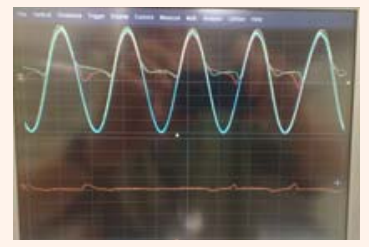

Figure 10: $\mathrm{V}_{\text {in }} @ 1 \mathrm{KHz}, 16 \mathrm{~V}_{\mathrm{p}-\mathrm{p}}$, $0^{\circ}$ phase difference

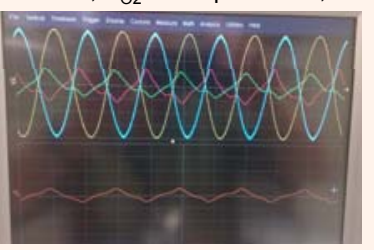

Figure 9: $\mathrm{V}_{\text {in }} @ 1 \mathrm{KHz}, 16 \mathrm{~V}_{\mathrm{p}-\mathrm{p}}$, $180^{\circ}$ phase difference

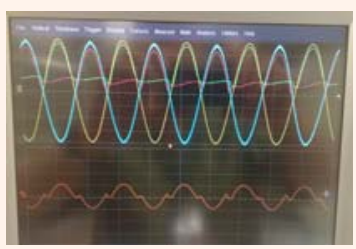

Figure 11: $\mathrm{V}_{\text {in }} @ 1 \mathrm{KHz}, 16 \mathrm{~V}_{\mathrm{p}-\mathrm{p}}$ $180^{\circ}$ phase difference

\section{CONCLUSION}

- The FeFET differential amplifier circuit produced some interesting results.

- Output signal characteristics changed with changes to input signal frequency and DC bias.

- The circuit operated over the tested frequency range of $100 \mathrm{~Hz}$ to $1 \mathrm{MHz}$.

- From Figures 8 and 10, the common-mode gain of the FeFET diff. amp. and the MOSFET diff. amp. are both approximately zero, as desired.

- From Figures 9 and 11, the difference-mode gain of the FeFET differential amplifier is less than the MOSFET differential amplifer.

\section{REFERENCES}

MacLeod, T. C. and Ho, F. D.:
Ferroelectrics. 2001; 34: 21-26.

2] MacLeod, Todd, C., A Study of the Characteristics of Ferroelectric Devices for use as Memory Circuits, Master's

Thesis, The University of Alabama in Huntsville, School of Graduate Studies, Huntsville, Alabama, 2007.

[3] Millman, Jacob, Microelectronics: Digital and Analog Circuits and Systems, New York: McGraw-Hill, 1979. 